\title{
Protective effect of extracorporeal membrane oxygenation on intestinal mucosal injury after cardiopulmonary resuscitation in pigs
}

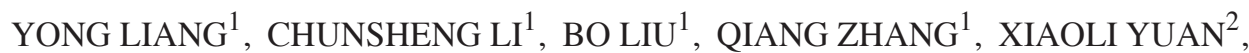 \\ YUN ZHANG $^{2}$, JIYANG LING ${ }^{2}$ and LIANXING ZHAO ${ }^{1}$
}

\author{
${ }^{1}$ Department of Emergency, Beijing Chao-Yang Hospital Affiliated to Capital Medical University, Beijing 100020; \\ ${ }^{2}$ Department of Emergency, Beijing Tong-Ren Hospital Affiliated to Capital Medical University, Beijing 100043, P.R. China
}

Received November 21, 2018; Accepted July 31, 2019

DOI: $10.3892 /$ etm.2019.8087

\begin{abstract}
The present study aimed to explore the protective effects of extracorporeal membrane oxygenation (ECMO) on intestinal mucosal injury following cardiopulmonary resuscitation (CPR), and to assess the potential mechanisms involved. A total of 24 healthy adult domestic pigs were selected as the study subjects. A ventricular fibrillation model was induced through programmed electric stimulation. Subsequently, the animals were randomly divided into conventional CPR and CPR+ECMO groups ( $\mathrm{n}=12$ per group). The mortality and hemodynamic parameters of the two groups were compared. The expression levels of inflammatory cytokines in the serum and intestinal mucosa were detected by ELISAs. The intestinal mucosa was subjected to hematoxylin and eosin, and immunohistochemical staining, followed by electron microscopy, to assess the degree of apoptosis and necrosis. The animals in both groups recovered from the programmed ventricular fibrillation. In the CPR group, two animals died at $2 \mathrm{~h}$ and two more animals died a further $2 \mathrm{~h}$ later, resulting in a $33.3 \%$ mortality rate, whereas no cases of mortality were observed in the CPR+ECMO group. Compared with the animals in the CPR group, the hemodynamic parameters of the animals in the CPR+ECMO group revealed significantly improved outcomes. Multiple inflammatory factors (tumor necrosis factor $\alpha$, interleukin-1 and interleukin-6), myeloperoxidase and malondialdehyde levels were decreased, whereas $\mathrm{Na} / \mathrm{Ca}$-ATPase and superoxide dismutase levels were elevated in the intestinal mucosa of animals in the CPR+ECMO group compared with those in the CPR group. Additionally, pathological
\end{abstract}

Correspondence to: Dr Chunsheng Li, Department of Emergency, Beijing Chao-Yang Hospital Affiliated to Capital Medical University, 8 Worker's Stadium South Road, Chao-Yang, Beijing 100020, P.R. China

E-mail: 1cscyyy@163.com

Key words: cardiac arrest, cardiopulmonary resuscitation, intestinal mucosal barrier, extracorporeal membrane oxygenation staining demonstrated that the intestinal mucosa tissue in the CPR+ECMO group exhibited less apoptosis, necrosis and inflammatory cell infiltration, which was further supported by a decrease in Bax expression and an increase in Bcl-2 expression. Overall, ECMO after CPR reduced the intestinal mucosal barrier injury after spontaneous circulation recovery, and the mechanism involved decreased inflammation and apoptosis.

\section{Introduction}

Post-cardiac arrest (CA) syndrome, or post-resuscitation multiple organ dysfunction syndrome (PR-MODS), has become an independent risk factor for the prognosis of patients with successful resuscitation after CA (1). The pathogenesis of PR-MODS has not been fully elucidated; however, it is currently considered that intestinal mucosal ischemia-reperfusion injury triggered by $\mathrm{CA}$ is an important factor leading to failure of multiple organs and tissues, and even mortality in patients following cardiopulmonary resuscitation (CPR) (1). The mortality rate of patients with PR-MODS after CA was as high as 60\% in the United states in 2015 (2). Current research on PR-MODS mainly focuses on the mechanism of myocardial and brain injury after CA. The treatment options include advanced respiratory cycle support and mild hypothermia for brain protection $(3,4)$. However, to the best of our knowledge, research associated with the pathophysiological alterations following CPR and corresponding prevention and treatment options remain lacking.

Extracorporeal membrane oxygenation (ECMO) is an extracorporeal technique of providing prolonged cardiac and respiratory support to injured heart and lungs $(5,6)$. This intervention has been widely applied in children, but it is also useful in adults with cardiac and respiratory failure $(7,8)$. The function of ECMO is to remove blood from the body of a patient and to artificially remove carbon dioxide and oxygenating red blood cells. ECMO is used either post-cardiopulmonary bypass or in late stage treatment of a person with profound heart and/or lung failure (9). This intervention is now recognized as a treatment for CA $(10,11)$, whereas its effects after CA and resuscitation on intestinal mucosal injury, and the potential mechanisms, to the best of our knowledge, have not been studied. 
Considering that ischemia-reperfusion injury is the main pathophysiological mechanism of intestinal injury following spontaneous CA (12), the present study proposed to correct the acute hypoxic state of the intestinal mucosa caused by hypoperfusion at the earliest time, which would help to block or reverse intestinal mucosal ischemia-reperfusion injury. The aim was to explore the protective effects of ECMO following CPR on intestinal mucosal injury, and to assess the potential mechanisms. The present findings may provide a basis for an important strategy to improve the prognosis of patients that have undergone CPR.

\section{Materials and methods}

Ethics statement. All experimental protocols followed in the present study were approved by the Ethics Committee of Capital Medical University (Beijing, China). The animals were kept in a pathogen-free environment and were given free access to drinking water and food. All procedures were performed strictly in accordance with the Institutional Animal Care Instructions.

Animal preparation, tracheal intubation and catheterization. Changbaishan domestic miniature swine purchased from Beijing Huaxia Xingyang Biological Technology Co., Ltd. [license no. SYXK (Beijing) 2017-0013] were used in the present study. A total of 24 healthy male pigs (age, 11-13 weeks; weight, $28.3 \pm 2.5 \mathrm{~kg}$ ) were selected and randomly divided into two groups: CPR group (treated with CPR after CA; $n=12$ ) and $\mathrm{CPR}+\mathrm{ECMO}$ group (treated with ECMO during CPR after $\mathrm{CA} ; \mathrm{n}=12$ ). Prior to the experimental procedures, all animals were fasted of solids overnight, followed by sedation using intramuscular injection with $10.0 \mathrm{mg} / \mathrm{kg}$ ketamine and $0.5 \mathrm{mg} / \mathrm{kg}$ midazolam. Subsequently, anesthesia was induced by ear vein injection with a bolus dose of $2.0 \mathrm{mg} / \mathrm{kg}$ propofol. The swine were then secured on the operating table for the subsequent procedures. All procedures were performed with aseptic surgical techniques at room temperature $\left(26^{\circ} \mathrm{C}\right)$, while the body temperature of the animals was maintained at $37^{\circ} \mathrm{C}$ under an infrared lamp. First, to maintain sufficient anesthetic and analgesic depth, boluses of anesthetics (1.0 mg/kg propofol) and analgesics $(4.0 \mu \mathrm{g} / \mathrm{kg}$ fentanyl) were administered intravenously. Propofol $(9.0 \mathrm{mg} / \mathrm{kg} / \mathrm{h})$ and fentanyl $(1.0 \mu \mathrm{g} / \mathrm{kg} / \mathrm{h})$ were delivered to maintain the level of anesthesia and analgesia according to physiological parameters, corneal and palpebral reflexes, and spontaneous movement. There was no difference in body weight or other characteristics, including the extra doses of propofol and fentanyl administered during the preparatory phase between the CPR and CPR+ECMO groups. At the end of the experiments ( $6 \mathrm{~h}$ after ROSC), potassium chloride $(74.5 \mathrm{mg} / \mathrm{kg})$, in conjunction with general anesthesia (9.0 mg/kg/h propofol and $1.0 \mu \mathrm{g} / \mathrm{kg} / \mathrm{h}$ fentanyl), was used to euthanize the animals.

The anesthetized animals were intubated with a $6.5-\mathrm{mm}$ cuffed endotracheal tube into the trachea via tracheotomy, and then mechanically ventilated with a volume-controlled ventilator (Draeger vi 400; Draegerwerk AG \& Co., KGaA) at a tidal volume of $15.0 \mathrm{ml} / \mathrm{kg}$ and a respiratory frequency of $12-20 / \mathrm{min}$ with an inspired oxygen fraction of $35 \%$ at the beginning. The end-tidal capnography $\left(\mathrm{CO}_{2} \mathrm{SMO}\right.$ Plus! ${ }^{\circledR}$ monitor; Respironics, Inc.) was used to monitor the partial pressure of carbon dioxide. Adequate baseline ventilation was determined using an arterial blood gas analyzer (ABL80; Radiometer Medical ApS).

Three surface electrodes were placed on the fore limbs of the animals to configure a standard lead II electrocardiogram (ECG) as previously described (13). The ECG was recorded using a multichannel physiological recorder (BL-420 F Data Acquisition and Analysis system; Chengdu TME Technology Co., Ltd.).

To induce ventricular fibrillation (VF) and maintain intravenous fluid therapy, a 5-Fr pacing catheter was placed into the right ventricle through the right external jugular vein. To measure the mean arterial pressure (MAP), a fluid-filled catheter (5 Fr; Terumo Corporation) was inserted into the aortic arch through the left femoral artery. A Swan-Ganz catheter (7 Fr; Edwards Lifesciences Corporation) was placed in the pulmonary artery through the left femoral vein to measure cardiac output (CO) using the thermodilution method (12). For animals in the ECMO group, a 14-Fr peripheral venous catheter (Dragon Laifu Medical Products Co., Ltd.) was additionally placed into the right atrium through the left internal jugular vein, as well as a 12-Fr artery catheter into the ascending aorta through the right femoral artery. All catheters were filled with heparinized normal saline solution $(5.0 \mathrm{U} / \mathrm{ml})$ to prevent clotting.

Hemodynamic parameters were monitored using an HP monitor (M1165; Hewlett-Packard Co.). Normal saline solution $(5.0 \mathrm{ml} / \mathrm{kg} / \mathrm{h})$ and colloidal fluid $(5.0 \mathrm{ml} / \mathrm{kg} / \mathrm{h})$ were infused intraoperatively to replenish fluid losses.

Extracorporeal life support. The ECMO circuit consisted of one arterial input catheter, one venous output catheter, a Levitronix CentriMag console (Levitronix $\mathrm{GmbH}$ ), a centrifugal pump head (Maquet Cardiopulmonary AG; Getinge AG) and a mechanical gas blender (Thoratec Corporation). The circuit was driven by the centrifugal pump head in line with the Adult Microporous Membrane Oxygenator with Bioline Coating (Maquet Cardiopulmonary AG).

The left internal jugular vein and the right femoral artery were cut. The ECMO circuit connected to the 16-Fr venous outflow cannula was placed in the left internal jugular vein and the 14-Fr arterial inflow cannula was placed in the right femoral artery. The catheters were filled with heparinized saline solution $(5.0 \mathrm{U} / \mathrm{ml})$ and clamped. The circuit was primed with colloid solution $(1,000 \mathrm{ml})$, preheparinized with $250 \mathrm{U} / \mathrm{kg}$ heparin, and had $50 \%$ oxygen air mix delivered to the oxygenator. The oxygen/air flow was repeatedly adjusted to maintain $\mathrm{pO}_{2}$ and $\mathrm{pCO}_{2}$ in the blood. A temperature of $37^{\circ} \mathrm{C}$ was maintained with a water-circulating heat exchanger. $\mathrm{VF}$ was induced by a programmed electrical stimulation instrument (GY-600A; Kaifeng Huanan Instrument Co.) with mode S1S2 (300/200 ms), $40 \mathrm{~V}, 8: 1$ proportion and -10-ms step length. VF was confirmed by the presence of a characteristic ECG tracing and an immediate drop in arterial blood pressure.

Experimental protocol. Animals were prepared according to the aforementioned procedure and the ECMO circuit was placed. Then, the animals were permitted to rest for $30 \mathrm{~min}$ to achieve a steady resting level and the baseline values of heart 
rate, MAP and CO were measured. Subsequently, VF was induced in all animals in the right ventricular apex until it was verified by the ECG trace, accompanied by a drop of arterial blood pressure. Mechanical ventilation was stopped when VF was induced successfully. After $12 \mathrm{~min}$, CPR was performed manually for $2 \mathrm{~min}$ in animals from the CPR+ECMO and CPR groups according to the 2015 guidelines for CPR (14). The depth of chest compression was $\sim 1 / 3$ of the anteroposterior diameter. A HeartstartMRx monitor/defibrillation with Q-CPR (Philips Medical Systems, Inc.) was used to ensure the quality of the compressions. A bag respirator with room air was used to ventilate the pigs, and the compression-to-ventilation ratio was 30:2. Subsequently, CPR was performed for $4 \mathrm{~min}$ followed by shock attempts in the CPR group as previously described $(15,16)$. After the shock attempts, CPR was continuously performed in the CPR group and CPR combined with ECMO was continuously performed in the CPR+ECMO group until the end of the experiment. Defibrillation was performed using diphase $4.0 \mathrm{~J} / \mathrm{kg}$ using a Heartstart M3535A defibrillator (Philips Medical Systems, Inc.) for the first attempt between the right infraclavicular electrode and the apical electrode. When necessary, additional epinephrine $(0.02 \mathrm{mg} / \mathrm{kg})$ was injected into the right atrium for the restoration of spontaneous circulation (ROSC).

The endpoint of the aforementioned experiments was $6 \mathrm{~h}$ after ROSC or the death of the animals in both groups. ROSC was considered to occur when MAP was $>60 \mathrm{mmHg}$ for $20 \mathrm{~min}$ or systolic blood pressure was $>80 \mathrm{mmHg}$. CPR was stopped in the CPR group when ROSC was achieved. Otherwise, CPR and defibrillation were performed alternately in animals in the CPR group. For the CPR+ECMO group, the flow rate of ECMO was maintained between 3 and $51 / \mathrm{min}$ to maintain MAP $>60 \mathrm{mmHg}$. To maintain the level of $\mathrm{pO}_{2}$ within 100-120 $\mathrm{mmHg}$, the oxygen flow was adjusted using the oxygenator. Animals were ventilated during the reperfusion of ECMO to keep end-tidal $\mathrm{pCO}_{2}$ within $35-45 \mathrm{mmHg}$ by adjusting the respiratory frequency. The activated clotting time was maintained at $>250 \mathrm{sec}$. CPR was considered to have failed if spontaneous circulation was not restored within $30 \mathrm{~min}$. Animals that were alive $6 \mathrm{~h}$ post-ROSC were sacrificed using potassium chloride $(74.5 \mathrm{mg} / \mathrm{kg})$, in conjunction with general anesthesia $(9.0 \mathrm{mg} / \mathrm{kg} / \mathrm{h}$ propofol and $1.0 \mu \mathrm{g} / \mathrm{kg} / \mathrm{h}$ fentanyl), administered intravenously.

Hemodynamic parameters. Hemodynamic parameters, including heart rate (HR), MAP and CO, were monitored. HR was recorded by the standard lead II ECG. MAP was measured with a fluid-filled catheter advanced from the left femoral artery into the aortic arch through a pressure transducer with pulse indicator continuous CO system. A Swan-Ganz catheter (7 Fr; Edwards Lifesciences Corporation) was advanced from the left femoral vein and flow directed into the pulmonary artery to measure $\mathrm{CO}$ by the thermodilution method (17). All values were recorded prior to VF for the baseline value, at ROSC (ROSC0), and various time points after ROSC, including 1 (ROSC1), 2 (ROSC2), 3 (ROSC3), 4 (ROSC4) and 6 h (ROSC6).

Serum and intestinal tissue acute injury biomarkers. Blood samples $(2.5 \mathrm{ml})$ were collected in $0.109 \mathrm{~mol} / 1$ trisodium citrate $(9: 1 \mathrm{v} / \mathrm{v})$ concomitantly at the following time points: Before VF (baseline), ROSC0, ROSC1, ROSC2, ROSC4 and
ROSC6. Blood samples were acquired from the left femoral by intravenous cannulation. The initial $5 \mathrm{ml}$ blood sample was discarded due to possible inclusion of heparinized normal saline solution. Subsequently, the samples were immediately centrifuged at $6,000 \mathrm{x}$ g for $10 \mathrm{~min}$ at $4^{\circ} \mathrm{C}$, and the supernatants were stored at $-80^{\circ} \mathrm{C}$ until further analysis.

The levels of interleukin (IL)-1 (cat. no. DY6226), IL-6 (cat. no. P6000B), tumor necrosis factor $\alpha$ (TNF- $\alpha$; cat. no. DY690B) in the blood samples as well as in the intestinal tissue homogenates were measured using an ELISA kit (R\&D Systems, Inc.) according to manufacturer's protocols.

Acute injury biomarkers in the blood samples and the intestinal tissue homogenates were detected using commercial analysis kits, according to the manufacturers' protocols. Primary analysis kits were used to measure the levels of malondialdehyde (MDA; cat. no. ab233471; Abcam), superoxide dismutase (SOD, cat. no. ab65354; Abcam) and myeloperoxidase (MPO; cat. no. ab105136, Abcam).

ELISA (Beijing Solarbio Science \& Technology Co., Ltd.) was used to measure the expression of $\mathrm{Na} / \mathrm{K}$-ATPase and Ca-ATPase (cat. nos. BC0065 and BC0965, respectively) in the intestinal mucosa homogenate.

Intestine histology examination. Samples from the intestine were fixed with $10 \%$ formalin at $4^{\circ} \mathrm{C}$ overnight, dehydrated and embedded in paraffin, cut into sections $(10 \mu \mathrm{m})$, stained with hematoxylin solution for $5 \mathrm{~min}$ and with eosin for $3 \mathrm{~min}$ at room temperature, and observed under a light microscope (magnification, 200x; CX41; Olympus Corporation).

Intestine ultrastructure examination. Fresh intestine tissues were cut into pieces $(1.0 \times 1.0 \times 1.0 \mathrm{~mm})$, treated with $3 \%$ glutaraldehyde at $4^{\circ} \mathrm{C}$ for $30 \mathrm{~min}$, flushed with PBS, fixed with $1 \%$ perosmic acid $\left(4^{\circ} \mathrm{C}, 30 \mathrm{~min}\right)$ and dehydrated with acetone. The tissues were infiltrated with a mixture of propylene oxide and epoxy resin overnight and embedded in resin. The tissues were then cut into serial ultrathin sections $(70 \mathrm{~nm})$ and stained with $4 \%$ uranyl acetate for $20 \mathrm{~min}$ followed by $0.5 \%$ lead citrate for $5 \mathrm{~min}$. The sections were examined using transmission electron microscopy (magnification, x10,000; JEM-1010; JEOL, Ltd.). The ultrastructure of the intestinal mucosal epithelial cells, including microvilli and mitochondria, was observed.

Intestine immunostaining. Immunostaining was performed on the fixed intestine tissues using a standard protocol with primary antibodies (18). Briefly, the intestine tissue was fixed in $10 \%$ formalin at $4^{\circ} \mathrm{C}$ overnight, embedded in paraffin and sliced into $5-\mu \mathrm{m}$ sections. The slides were deparaffinized and incubated with $20.0 \mathrm{mg} / \mathrm{ml}$ proteinase $\mathrm{K}$ for $15 \mathrm{~min}$ at $4^{\circ} \mathrm{C}$. Endogenous peroxidase activity was inhibited using $3 \%$ hydrogen peroxide at room temperature for $10 \mathrm{~min}$. The slides were incubated with the primary antibodies, including monoclonal anti-Bax and anti-Bcl-2 antibodies (both 1:10,000 dilution; Bcl-2: cat. no. 2870; Bax, cat. no. 5023; Cell Signaling Technology, Inc.) at $4^{\circ} \mathrm{C}$ overnight followed by incubation with the secondary horseradish peroxidase-conjugated goat anti-rabbit IgG antibody $(1: 1,000$ dilution; cat. no. A16104SAMPLE; Thermo Fisher Scientific, Inc.). The staining results were observed under a light microscope (magnification, x200; CX41; Olympus Corporation). 
Two investigators blinded to the groups semi-quantitatively scored the slides by evaluating the staining intensity in four fields of each image as described (18).

TUNEL assay. Apoptosis was examined using a TUNEL apoptosis detection kit (EMD Millipore), according to the manufacturer's protocol. In brief, the intestine tissue was fixed in $10 \%$ formalin at $4^{\circ} \mathrm{C}$ overnight, embedded in paraffin and sliced into $5-\mu \mathrm{m}$ sections. The slides were deparaffinized, rehydrated and incubated with $20.0 \mathrm{mg} / \mathrm{ml}$ proteinase $\mathrm{K}$ for $15 \mathrm{~min}$ at $4^{\circ} \mathrm{C}$. Endogenous peroxidase activity was inhibited using 3\% hydrogen peroxide. Sections were then incubated with terminal deoxynucleotidyl transferase enzyme at $37^{\circ} \mathrm{C}$ for $1 \mathrm{~h}$. Staining was revealed using the 3,3-diaminobenzidine chromogen and the nucleus was counterstained by hematoxylin for $5 \mathrm{~min}$ at room temperature. Mounting Medium (cat. no. ab64230; Abcam) was applied to mount the slide and the staining was observed under a light microscope (CX41; Olympus Corporation). Four fields in each slide were randomly analyzed (magnification, x200). The grey value was analyzed using ImageProPlus software (version 7.0; Media Cybernetics, Inc.) as previously described (18). At $\geq 4$ fields of view were randomly selected from each slide and analyzed.

Statistical analysis. Statistical analysis was performed using GraphPad Prism software (version 5; GraphPad Software, Inc.). All quantitative data are expressed as the mean $\pm \mathrm{SD}$ from 12 experimental repeats. Comparisons between two groups of quantitative data were performed by Student's t-test when both sets of data fit the normal distribution and the homogeneity of variance was accepted. A non-parametric Mann-Whitney test was used when any set of data did not fit the normal distribution. Comparisons among multiple groups of quantitative data were performed using repeated one-way ANOVA when the data fit normal distribution, followed by rank-sum test (Mann-Whitney rank) and Bonferroni post hoc test. Log-rank (Mantel-Cox) test was used to analyze survival rates. $\mathrm{P}<0.05$ was considered to indicate a statistically significant difference.

\section{Results}

Basic characteristics and variables of the animals. No significant differences were identified in body length, body weight, dose of anesthetics and animal preparation time between the two groups $(\mathrm{P}>0.05$; Table I).

$C P R+E C M O$ treatment and mortality. As shown in Fig. 1, two pigs died $2 \mathrm{~h}$ after CPR in the CPR group due to irreversibly decreased blood pressure and blood oxygen. At $4 \mathrm{~h}$, two more pigs died. The overall mortality rate in the CPR group was $33.3 \%$. On the other hand, no rapid mortality was observed in the CPR+ECMO group. The $6 \mathrm{~h}$-survival rate was $100 \%$, suggesting that ECMO may improve the prognosis of animals with CA.

ECMO treatment improves hemodynamic parameters. The basal HR and $\mathrm{CO}$ of the animals in the two groups were not significantly different (CPR vs. CPR+ECMO; HR, 119.2 \pm 6.7 vs. $121.1 \pm 6.4 \mathrm{bpm} ; \mathrm{CO}, 3.7 \pm 0.5$ vs. $4.0 \pm 0.8 \mathrm{l} / \mathrm{min}$; both $\mathrm{P}>0.05$; Fig. 2). When compared with that in the CPR group, the HR
Table I. Variables of the animals in the CPR and CPR+ECMO groups.

\begin{tabular}{lccc}
\hline Index & $\begin{array}{c}\text { CPR } \\
\text { group }\end{array}$ & $\begin{array}{c}\text { CPR+ECMO } \\
\text { group }\end{array}$ & P-value \\
\hline Body length, $\mathrm{cm}$ & $107.2 \pm 2.9$ & $105.8 \pm 3.6$ & 0.3 \\
Body weight, $\mathrm{kg}$ & $34.17 \pm 4.4$ & $35.42 \pm 3.2$ & 0.4 \\
Propofol, $\mathrm{mg}$ & $202.8 \pm 3.9$ & $203.9 \pm 4.5$ & 0.5 \\
Fentanyl, $\mu \mathrm{g}$ & $125.4 \pm 6.2$ & $130.2 \pm 11.7$ & 0.2 \\
Preparation time, min & $135.4 \pm 10.3$ & $135.4 \pm 13.7$ & 1.0 \\
\hline
\end{tabular}

CPR, cardiopulmonary resuscitation; ECMO, extracorporeal membrane oxygenation.

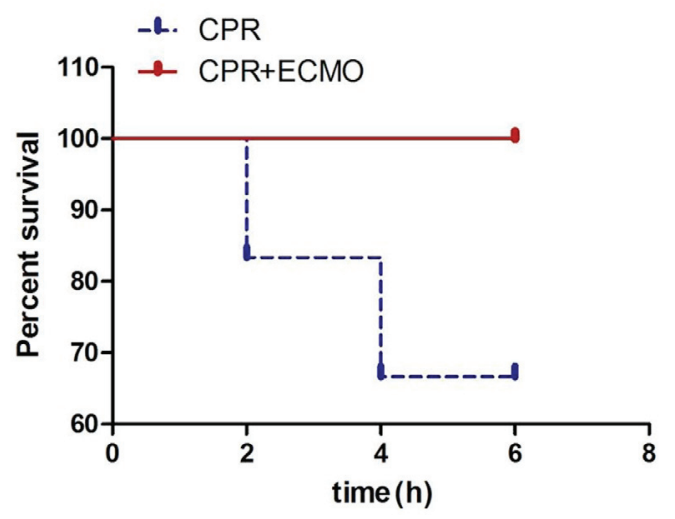

Figure 1. Survival curves of animals, in terms of hours after ROSC, of CPR and CPR+ECMO groups. The survival rate was $66.7 \%$ at the 6 -h time point in the CPR group, whereas no cases of mortality were recorded in the CPR+ECMO group. The survival rate was $100 \%$ after $6 \mathrm{~h}$ in the CPR+ECMO group. The survival curves were statistically different according to a log-rank (Mantel-Cox) test $(\mathrm{P}=0.0325)$. CPR, cardiopulmonary resuscitation; ECMO, extracorporeal membrane oxygenation.

of the animals in the CPR+ECMO group was significantly decreased at $0,1,2,3,4$ and $6 \mathrm{~h}$ after successful resuscitation (all $\mathrm{P}<0.01$ ), and $\mathrm{CO}$ was increased significantly at each time point (all $\mathrm{P}<0.01$ ).

ECMO decreases the levels of inflammatory factors in the circulation and intestinal mucosa. On a tissue level, compared with levels in the CPR group, TNF- $\alpha$, IL-1, IL-6 and MPO levels in the tissue homogenates of mucosa specimens $6 \mathrm{~h}$ after ROSC were significantly decreased in the CPR+ECMO group, whereas the levels of $\mathrm{Na}^{+} / \mathrm{Ca}^{2+}$-ATPase in the tissue were significantly increased (Fig. 3A-F). On a serum level, compared with those recorded in the CPR group, the levels of TNF- $\alpha$, IL- 1 and IL- 6 measured in the serum of animals from the CPR+ECMO group appeared to be decreased, though the difference was not statistically significant (Fig. 3G-I).

ECMO enhances SOD and decreases MDA in intestinal mисоsa. Compared with those in the CPR group, the SOD levels were significantly higher in the CPR+ECMO group, whereas the levels of tissue MDA were significantly lower (Fig. 4). 


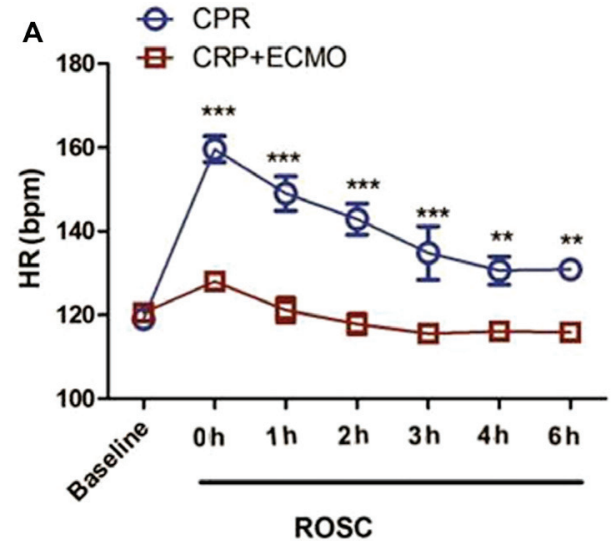

B

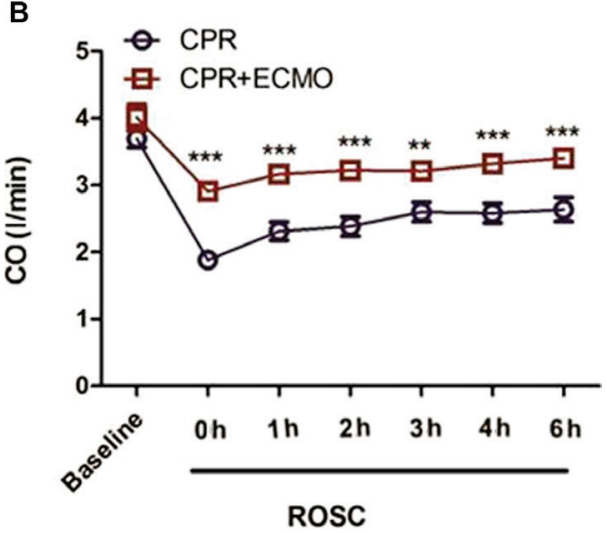

Figure 2. Comparison of hemodynamic parameters between the two groups. (A) Comparison of ventricular rate between animals from the CPR and the CPR+ECMO groups at baseline and at $0,1,2,3,4$ and $6 \mathrm{~h}$ after successful resuscitation. (B) $\mathrm{CO}$ was compared between animals from the CPR and CPR+ECMO groups at baseline, as well as $0,1,2,3,4$ and $6 \mathrm{~h}$ after successful resuscitation. Data at each time point are presented as the mean $\pm \mathrm{SD}$. $\mathrm{n}=12$ in both groups. ${ }^{* * *} \mathrm{P}<0.01$ and ${ }^{* * * *} \mathrm{P}<0.001$ vs. $\mathrm{CPR}$. CO, cardiac output; CPR, cardiopulmonary resuscitation; ECMO, extracorporeal membrane oxygenation; HR, heart rate; ROSC, restoration of spontaneous circulation.

ECMO reduces intestinal mucosal epithelial barrier damage. HE staining demonstrated that the intestinal mucosal epithelial cells in the CPR+ECMO group exhibited significantly reduced congestion and edema compared with those in the CPR group. The mucosal barrier was almost intact in the CPR+ECMO group, and the infiltration of sub-epithelial inflammatory cells was significantly reduced (Fig. 5A), suggesting that ECMO can reduce intestinal epithelial mucosal barrier damage. TUNEL staining revealed that the number of TUNEL-positive cells in the intestinal mucosal epithelium of the CPR+ECMO group was significantly lower than that of the CPR group (Fig. 5B and E), suggesting that ECMO treatment can inhibit intestinal epithelial cell apoptosis after CA. In addition, immunohistochemistry revealed that the expression levels of anti-apoptotic protein $\mathrm{Bcl}-2$ in the intestinal mucosa of the CPR+ECMO treatment group were higher than those in the CPR group, whereas the expression of apoptosis-associated protein Bax was significantly lower than that in the CPR group (Fig. 5C-E). This further supports the hypothesis that ECMO treatment inhibits apoptosis in intestinal mucosal epithelial cells. Finally, electron microscopy revealed that the degree of apoptosis and necrosis of the intestinal mucosa in the CPR+ECMO group was markedly lower than that in the CPR group (Fig. 6).

\section{Discussion}

The present study induced VF in pigs and examined whether CPR+ECMO could help improve CA outcome compared with regular CPR. The results demonstrated that ECMO could significantly reduce acute mortality after $\mathrm{CA}$, and improve the hemodynamic parameters. Pathophysiological results revealed that the intestinal mucosa of the CPR group exhibited obvious congestion and edema, cell shedding and villous necrosis at $6 \mathrm{~h}$ after resuscitation.

Patients who have suffered CA have ineffective circulatory blood pressure, and their intestines are in a non-perfused state $(19,20)$. A previous study reported that a 4-min CA can lead to intestinal hypoperfusion for up to $60 \mathrm{~min}$ (21). Due to insufficient effective perfusion, the intestinal tissue is severely ischemic and hypoxic, resulting in ischemic and hypoxic damage to the epithelial villus of the intestinal mucosa, which leads to intestinal mucosal epithelial edema, cell necrosis, shedding, and even complete mucosal shedding and ulceration (22). In addition to intestinal mucosal damage caused by direct ischemia and hypoxia, ischemia-reperfusion injury is an important pathophysiological mechanism of intestinal mucosal injury following CA (23). When ischemia-reperfusion occurs, intestinal mucosa tissue may be enriched with inflammatory cells that secrete a large number of inflammatory mediators, cytokines, toxins, oxygen free radicals, endotoxins and intestinal bacteria into the bloodstream, which eventually leads to apoptosis and multiple organ failure (24). ECMO is an emerging in vitro life-support system that allows the body to perform extracorporeal blood circulation and effective gas exchange in the absence of its own cardiopulmonary circulation, thereby maintaining the effective perfusion and oxygenation of organs (25). Therefore, ECMO has been used for acute breathing for patients with acute respiratory distress syndrome, cardiopulmonary failure, sepsis and cardiogenic shock $(26,27)$. The results of the present study suggest that early CPR with ECMO treatment can quickly halt the acute ischemia and hypoxia in the intestinal mucosa, improving the prognosis of patients. Furthermore, ECMO can block intestinal ischemia-reperfusion injury in CA model animals to some extent. Propofol has been reported to exhibit an anti-arrhythmic effect $(28,29)$. In the present study, the difference between the dosage of propofol used in the two groups of animals was not statistically significant. Thus, the dosage administered in the two groups was comparable. It can be concluded that the influence of propofol in the two groups of animals was similar, or that the effect was not statistically different even if propofol had an anti-arrhythmic effect. Therefore, the dosage of propofol would not have affected the mortality or other results between the two groups.

Currently, the majority of studies in the field of CPR treatment focus on whether hypothermia treatment can improve intestinal mucosal barrier damage $(30,31)$. However, the mechanism of intestinal mucosal injury after CA is not fully understood and, therefore, research on associated interventions is still in progress (32). Preventing acute ischemia and hypoxia as well as acute ischemia-reperfusion injury is fundamental to prevent intestinal mucosal barrier damage after CPR and to prevent PR-MODS (33). The present study revealed that timely initiation of ECMO treatment after CA could significantly 

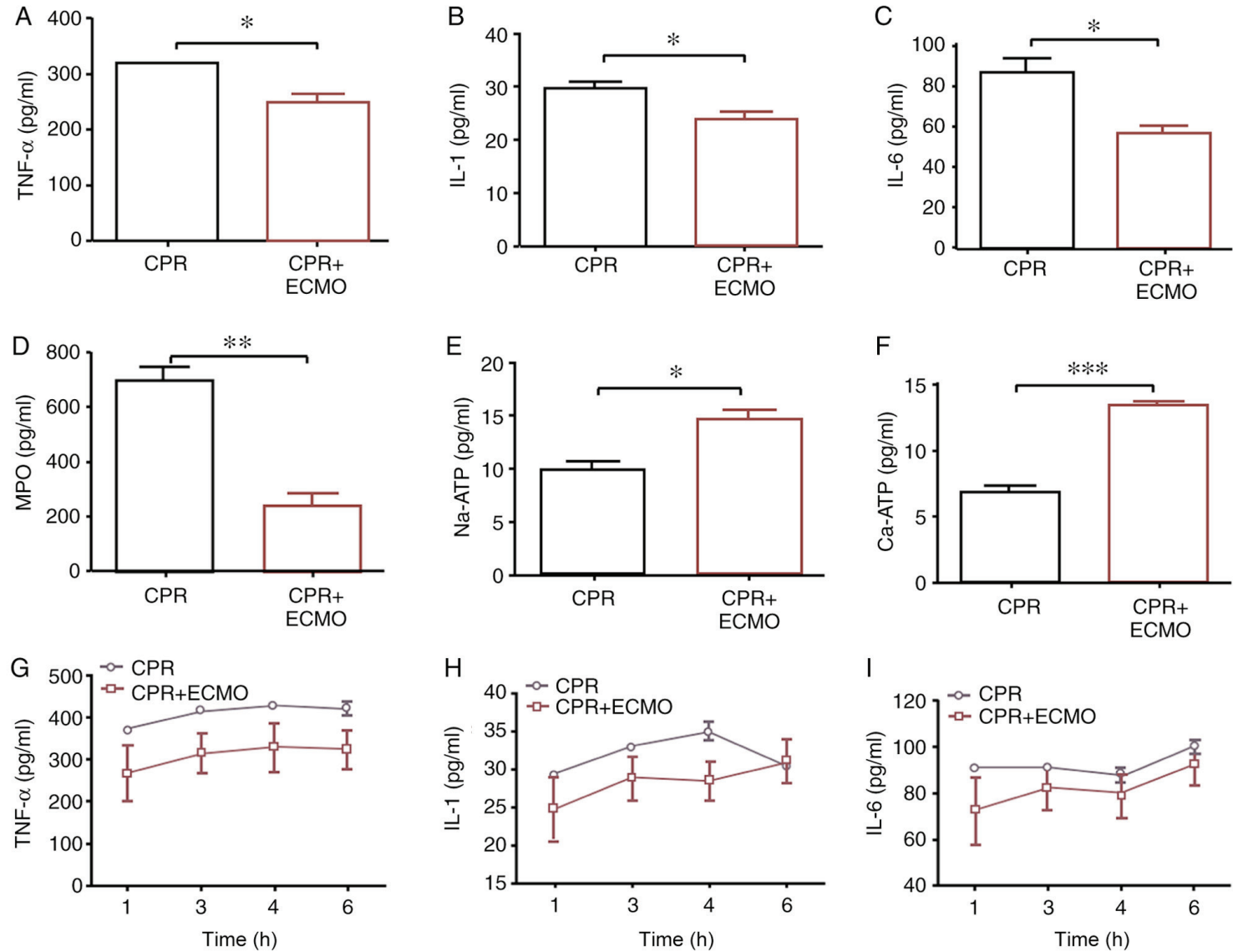

Figure 3. Comparison of expression levels of inflammation-associated mediators in circulation or intestinal mucosa between the two groups. Expression levels of (A) tissue TNF- $\alpha$, (B) tissue IL-1, (C) tissue IL-6, (D) tissue MPO, (E) tissue Na-ATP and (F) tissue Ca-ATP, and (G) serum TNF- $\alpha$, (H) serum IL-1 and (I) serum IL-6. Data are expressed as the mean \pm SD. $n=12$. " $\mathrm{P}<0.05 ;{ }^{* *} \mathrm{P}<0.01 ;{ }^{* * *} \mathrm{P}<0.001$. CPR, cardiopulmonary resuscitation; ECMO, extracorporeal membrane oxygenation; IL, interleukin; MPO, myeloperoxidase; TNF- $\alpha$, tumor necrosis factor $\alpha$.
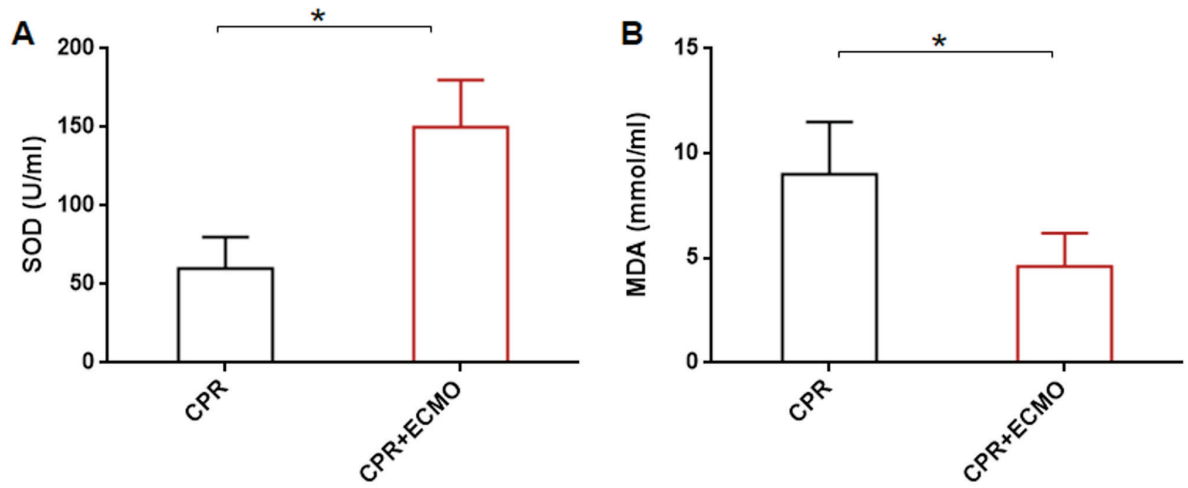

Figure 4. Comparison of levels of SOD and MDA in intestinal mucosa tissues from the two groups. Expression levels of (A) SOD and (B) MDA. Data are expressed as the mean $\pm \mathrm{SD}$. $\mathrm{n}=8-12$. "P<0.05. CPR, cardiopulmonary resuscitation; ECMO, extracorporeal membrane oxygenation; MDA, malondialdehyde; SOD, superoxide dismutase.

improve hemodynamic disorders, restore tissue perfusion and oxygen supply, correct hypoxemia, reduce the systemic inflammatory response and the oxidative stress response, and thereby reduce the ischemia-reperfusion injury of intestinal mucosa and the incidence and mortality of PR-MODS. The results suggest that ECMO treatment can effectively prevent PR-MODS from occurring in model animals after CPR in the acute phase of CA. However, it remains unclear whether
ECMO can improve the prognosis of patients after CA and resuscitation. Furthermore, the long-term efficacy of ECMO and the mechanisms of reducing the systemic inflammatory response and intestinal mucosal barrier damage require additional investigation.

In the animals in the CPR group, the expression levels of MPO in circulating plasma and the intestinal mucosa were significantly increased, suggesting an increase in neutrophil 


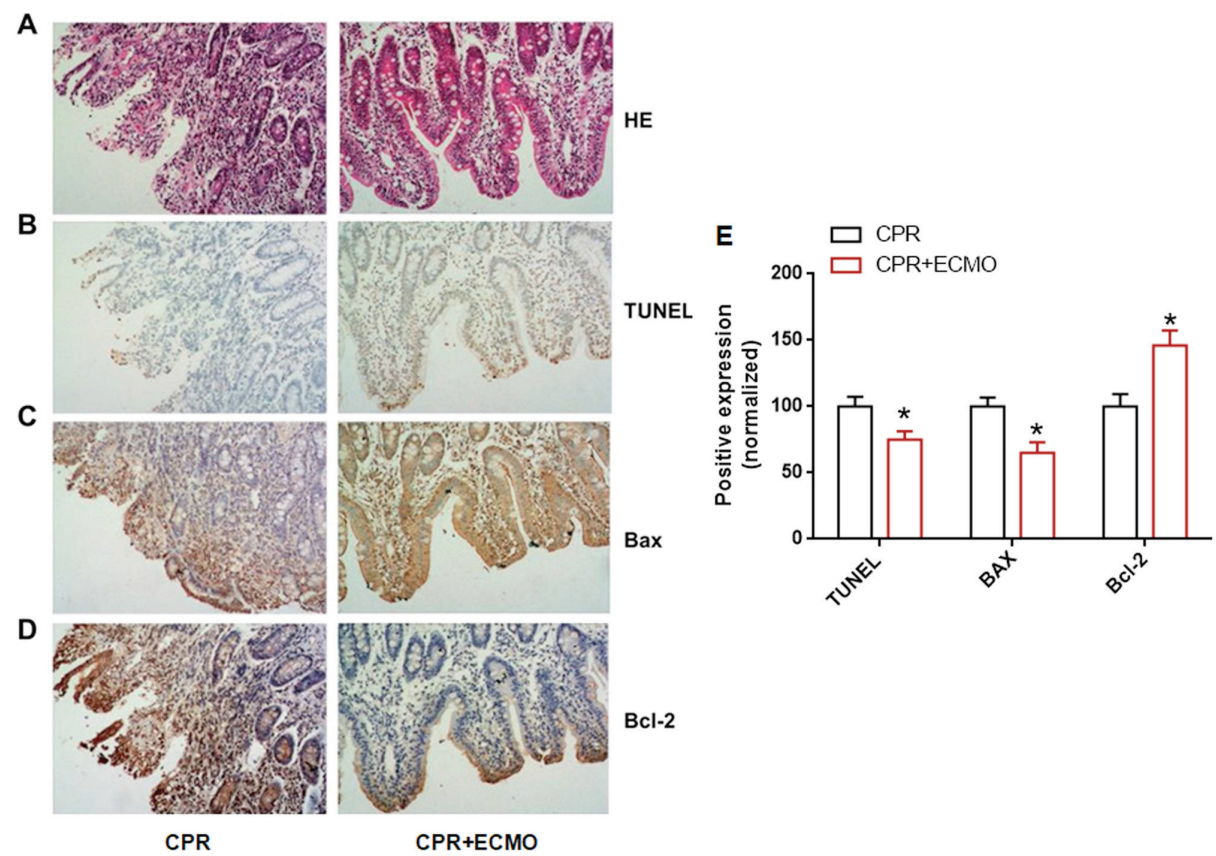

Figure 5. Evaluation of intestinal mucosal injury and apoptosis in the two groups of animals. (A) HE, (B) TUNEL, (C) Bax and (D) Bcl-2 staining of samples from the CPR and CPR+ECMO groups. (E) Semi-quantification of the TUNEL, Bax and Bcl-2 staining. Magnification, x200. "P<0.05 vs. CPR group. CPR, cardiopulmonary resuscitation; ECMO, extracorporeal membrane oxygenation; HE, hematoxylin and eosin.
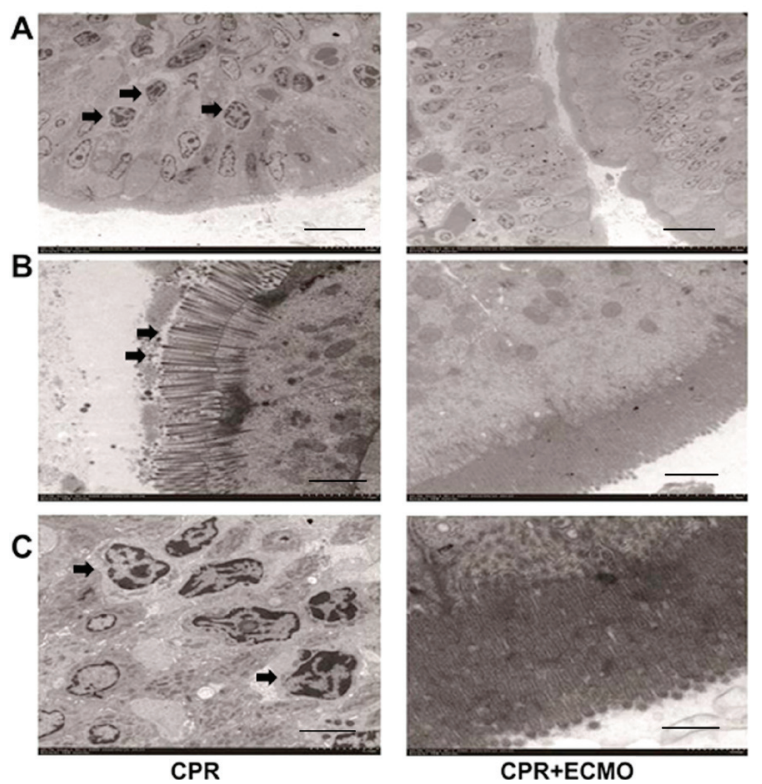

Figure 6. Electron microscopy results of intestinal mucosa tissues from animals in the two groups. (A) Representative electron microscopy images of intestinal mucosa specimens from the CPR group (left panels) and the CPR+ECMO group (right panels). The intestinal diffuse epithelial cells of intestinal mucosa in CPR group had severe degeneration and necrosis, basement membrane rupture, unclear demarcation, lysosome increase, (B) cell gap enlargement, tight junction disappearance, and breakdown of microvilli on the surface of epithelial cells. (C) The nuclei of epithelial cells were concentrated, chromatin edges were gathered, and the nuclear membrane was invaded to form apoptotic bodies, showing apoptotic state in CPR group Arrows indicate injury of the tissue. Scale bar, $5 \mu \mathrm{m}$. CPR, cardiopulmonary resuscitation; ECMO, extracorporeal membrane oxygenation.

infiltration (34). The inflammatory mediators (TNF- $\alpha$, IL-1 and IL-6) and acute phase-associated protein (MPO) were significantly upregulated, suggesting that 'inflammation storms' occurred systemically and locally (35). Overall, the intestinal mucosal epithelial cells in CPR animals experienced excessive apoptosis and the mucosal barrier was destructed. The timely treatment with ECMO after CA significantly improved the circulation of the animals and prevented inflammatory injury in the intestinal mucosa, characterized by decreased neutrophil infiltration, decreased inflammatory mediators, decreased cytokine expression, and reduced apoptosis of intestinal mucosal epithelial cells. As previously reported, SOD and MDA are opposing factors of cell survival (36). SOD has a protective role, while MDA has the opposite effect (36). The present study demonstrated that ECMO increased the SOD level and reduced the MDA level. These data reveal the protective effect of ECMO.

The intestinal mucosal epithelial layer is composed of a single layer of columnar epithelial cells, including absorbing, goblet and Paneth cells. The normal intestinal mucosal barrier is composed of heterogeneous elements, including a mechanical barrier that is made of tight junctions between the absorbed cells, an immunological barrier composed of defensing and lysozyme secreted by Paneth cells, and a chemical barrier composed of mucus secreted by the goblet cells (37). In this present study, typical morphological changes were observed in CPR group according to the images obtained from TEM, but not in those from the CPR+ECMO group.

The present study had several additional limitations. First, the physiology of the swine model may not reflect the situation in patients suffering from $\mathrm{CA}$, and the results should be confirmed in clinical practice. Secondly, all the catheters of ECMO circuits were placed in advance; however, in the clinical practice, there exists some difficulty in cannulating. Thirdly, the experimental endpoint was $6 \mathrm{~h}$ after ROSC and the observation duration was relatively short. Fourthly, miniature swine were selected in the present study. The animal size may also affect the outcomes of 
CPR and ECMO, which may cause bias. Finally, the sample size in the present study was limited and a study with a larger sample size should be performed in the future.

In conclusion, ECMO treatment may correct intestinal mucosal ischemia-reperfusion injury in a timely manner, reduce the incidence of PR-MODS after CPR in CA, and thereby improve the prognosis of patients and reduce acute mortality.

\section{Acknowledgements}

Not applicable.

\section{Funding}

The study was supported by the National Natural Science Foundation of China (grant no. 81372025).

\section{Availability of data and materials}

The datasets used and/or analyzed during the current study are available from the corresponding author on reasonable request.

\section{Authors' contributions}

YL, CSL, BL, QZ, XY, YZ, JL and LZ performed the experiments and analyzed the data. YL and LZ designed the study and wrote the manuscript.

\section{Ethics approval and consent to participate}

All experimental protocols followed in the present study were approved by the Ethics Committee of Capital Medical University (Beijing, China). All procedures were performed strictly in accordance with the Institutional Animal Care Instructions.

\section{Patient consent for publication}

Not applicable.

\section{Competing interests}

The authors declare that they have no competing interests.

\section{References}

1. Mentzelopoulos SD and Zakynthinos SG: Post-cardiac arrest syndrome: Pathological processes, biomarkers and vasopressor support, and potential therapeutic targets. Resuscitation 121: A12-A14, 2017.

2. Callaway CW, Donnino MW, Fink EL, Geocadin RG, Golan E, Kern KB, Leary M, Meurer WJ, Peberdy MA, Thompson TM and Zimmerman JL: Part 8: Post-cardiac arrest care: 2015 American heart association guidelines update for cardiopulmonary resuscitation and emergency cardiovascular care. Circulation 132 (18 Suppl 2): S465-S482, 2015.

3. Perman SM, Grossestreuer AV, Wiebe DJ, Carr BG, Abella BS and Gaieski DF: The utility of therapeutic hypothermia for post-cardiac arrest syndrome patients with an initial nonshockable rhythm. Circulation 132: 2146-2151, 2015.

4. Jahandiez V, Cour M, Bochaton T, Abrial M, Loufouat J, Gharib A, Varennes A, Ovize M and Argaud L: Fast therapeutic hypothermia prevents post-cardiac arrest syndrome through cyclophilin D-mediated mitochondrial permeability transition inhibition. Basic Res Cardiol 112: 35, 2017.
5. Bacon MK, Gray SB, Schwartz SM and Cooper DS: Extracorporeal membrane oxygenation (ECMO) support in special patient populations-the bidirectional glenn and fontan circulations. Front Pediatr 6: 299, 2018.

6. Wu XY, Zhuang ZQ, Zheng RQ and Liu SQ: Extracorporeal membrane oxygenation as salvage therapy for acute massive pulmonary embolism after surgery for tibiofibular fractures. Chin Med J (Engl) 131: 2611-2613, 2018.

7. Hernandez Conte AT, Ng D, Ramzy D, Dilibero D, LaBounty TM, Gaultier C and Behringer EC: Extracorporeal membrane oxygenation in a 29-year-old man with Pneumocystis jirovecii respiratory failure and AIDS. Tex Heart Inst J 45: 254-259, 2018.

8. Kim H, Paek JH, Song JH, Lee H, Jhee JH, Park S, Yun HR, Kee YK, Han SH, Yoo TH, et al: Permissive fluid volume in adult patients undergoing extracorporeal membrane oxygenation treatment. Crit Care 22: 270, 2018.

9. Panda BR, Prabhu A, Provenzano S and Karl T: Use of extracorporeal life support for emergency coronary artery bypass grafting. Interact Cardiovasc Thorac Surg 16: 897-899, 2013.

10. Tan BK: Extracorporeal membrane oxygenation in cardiac arrest. Singapore Med J 58: 446-448, 2017.

11. Conrad SA and Rycus PT: Extracorporeal membrane oxygenation for refractory cardiac arrest. Ann Card Anaesth 20 (Suppl 1): S4-S10, 2017.

12. Pan H, Chen D, Liu B, Xie X, Zhang J and Yang G: Effects of sodium hydrosulfide on intestinal mucosal injury in a rat model of cardiac arrest and cardiopulmonary resuscitation. Life Sci 93: 24-29, 2013.

13. Meek S and Morris F: ABC of clinical electrocardiography. Introduction. I-Leads, rate, rhythm, and cardiac axis. BMJ 324: 415-418, 2002.

14. Maudet L, Carron PN and Trueb L: Cardiopulmonary resuscitation: The essential of 2015 guidelines. Rev Med Suisse 12: 313-317, 2016 (In French)

15. Hang CC, Li CS, Wu CJ and Yang J: Acute kidney injury after cardiac arrest of ventricular fibrillation and asphyxiation swine model. Am J Emerg Med 32: 208-215, 2014.

16. Wu CJ, Li CS, Zhang Y and Yang J: Application of positron emission tomography in the detection of myocardial metabolism in pig ventricular fibrillation and asphyxiation cardiac arrest models after resuscitation. Biomed Environ Sci 27: 531-536, 2014.

17. Gawlinski A: Measuring cardiac output: Intermittent bolus thermodilution method. Crit Care Nurse 20: 118-120, 122-114, 2000.

18. Chen Y, Huang Y, Lu X, Wang G and Chi P: Antitumor effects of the silencing of programmed cell death ligand 1 in colorectal cancer via immunoregulation. Oncol Rep 40: 3370-3380, 2018.

19. MacFie J: Enteral versus parenteral nutrition: The significance of bacterial translocation and gut-barrier function. Nutrition 16: 606-611, 2000.

20. Schroeder DC, Maul AC, Mahabir E, Koxholt I, Yan X, Padosch SA, Herff H, Bultmann-Mellin I, Sterner-Kock A, Annecke T, et al: Evaluation of small intestinal damage in a rat model of 6 Minutes cardiac arrest. BMC Anesthesiol 18: 61, 2018.

21. Wurm R, Cho A, Arfsten H, van Tulder R, Wallmüller C, Steininger P, Sterz F, Tendl K, Balassy C, Distelmaier K, et al: Non-occlusive mesenteric ischaemia in out of hospital cardiac arrest survivors. Eur Heart J Acute Cardiovasc Care 7: 450-458, 2018.

22. Korth U, Krieter H, Denz C, Janke C, Ellinger K, Bertsch T, Henn $\mathrm{C}$ and Klein J: Intestinal ischaemia during cardiac arrest and resuscitation: Comparative analysis of extracellular metabolites by microdialysis. Resuscitation 58: 209-217, 2003.

23. Patil KD, Halperin HR and Becker LB: Cardiac arrest: Resuscitation and reperfusion. Circ Res 116: 2041-2049, 2015.

24. Parks DA and Granger DN: Contributions of ischemia and reperfusion to mucosal lesion formation. Am J Physiol 250: G749-G753, 1986.

25. Millar JE, Fanning JP, McDonald CI, McAuley DF and Fraser JF: The inflammatory response to extracorporeal membrane oxygenation (ECMO): A review of the pathophysiology. Crit Care 20: 387,2016

26. Tu Y, Jin Q, Sun R and Li Q: Extracorporeal membrane oxygenation support for a multitrauma patient with ARDS: A case report and literature review. Exp Ther Med 15: 2062-2065, 2018.

27. Tillmann BW, Klingel ML, Iansavichene AE, Ball IM and Nagpal AD: Extracorporeal membrane oxygenation (ECMO) as a treatment strategy for severe acute respiratory distress syndrome (ARDS) in the low tidal volume era: A systematic review. J Crit Care 41: 64-71, 2017 
28. Liu Q, Kong AL, Chen R, Qian C, Liu SW, Sun BG, Wang LX, Song LS and Hong J: Propofol and arrhythmias: Two sides of the coin. Acta Pharmacol Sin 32: 817-823, 2011.

29. Warpechowski P, dos Santos AT, Pereira PJ and de Lima GG: Effects of propofol on the cardiac conduction system. Rev Bras Anestesiol 60: 438-444, 2010 (In English, Portuguese).

30. Van Hauwermeiren F, Vandenbroucke RE, Grine L, Lodens $S$, Van Wonterghem E, De Rycke R, De Geest N, Hassan B and Libert C: TNFR1-induced lethal inflammation is mediated by goblet and Paneth cell dysfunction. Mucosal Immunol 8 : 828-840, 2015.

31. Xiong W, Xu S, Li H and Liang K: Moderate hypothermia ameliorates enterocyte mitochondrial dysfunction in severe shock and reperfusion. J Surg Res 200: 250-259, 2016.

32. Stub D, Bernard S, Duffy SJ and Kaye DM: Post cardiac arrest syndrome: A review of therapeutic strategies. Circulation 123: 1428-1435, 2011.

33. de Oliveira THC, Souza DG, Teixeira MM and Amaral FA: Tissue dependent role of PTX3 during ischemia-reperfusion injury. Front Immunol 10: 1461, 2019.
34. Lowe PP, Gyongyosi B, Satishchandran A, Iracheta-Vellve A, Ambade A, Kodys K, Catalano D, Ward DV and Szabo G: Alcohol-related changes in the intestinal microbiome influence neutrophil infiltration, inflammation and steatosis in early alcoholic hepatitis in mice. PLoS One 12: e 0174544, 2017.

35. D'Elia RV, Harrison K, Oyston PC, Lukaszewski RA and Clark GC: Targeting the 'cytokine storm' for therapeutic benefit. Clin Vaccine Immunol 20: 319-327, 2013.

36. Zhu G, Wang X, Wu S, Li X and Li Q: Neuroprotective effects of puerarin on 1-methyl-4-phenyl-1,2,3,6-tetrahydropyridine induced Parkinson's disease model in mice. Phytother Res 28: 179-186, 2014.

37. Martens EC, Neumann M and Desai MS: Interactions of commensal and pathogenic microorganisms with the intestinal mucosal barrier. Nat Rev Microbiol 16: 457-470, 2018.

This work is licensed under a Creative Commons Attribution-NonCommercial-NoDerivatives 4.0 International (CC BY-NC-ND 4.0) License. 